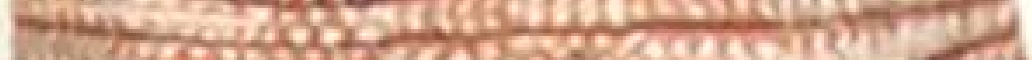




\section{AUTOESTIMA Y FUNCIONALIDAD FAMILIAR EN ESTUDIANTES DE BACHILLERATO}

SELF-ESTEEM AND FAMILY FUNCTIONALITY IN HIGH SCHOOL STUDENTS

\section{MARÍA LETICIA SEGURA ARÉVALO MARÍA ELENA URDIALES IBARRA}

\section{RESUMEN}

Escuela y familia son un binomio vinculado con el rendimiento académico y la salud física y emocional de los adolescentes. En la escuela es donde toman gran influencia de sus pares redituando en la personalidad de los estudiantes, por lo que la autoestima cobra gran impacto como fenómeno psicológico. Por otro lado, en la familia es donde se establecen los primeros vínculos afectivos influyendo grandemente la forma en que los adolescentes conviven y se relacionan. El objetivo del presente estudio es describir la relación entre autoestima y funcionalidad familiar en adolescentes de bachillerato. Se realizó un estudio de tipo descriptivo para evaluar la autoestima y funcionalidad familiar de 349 estudiantes de 2 do semestre de una preparatoria pública al poniente del área metropolitana de Monterrey. Se utilizó la escala de autoestima Forma-5 (AF5) y el cuestionario Family APGAR con apoyo de la plataforma google forms. La aplicación fue colectiva dentro del recinto universitario con previa autorización y consentimiento. Los resultados revelan en la mayoría de los adolescentes funcionalidad familiar, además de correlación significativa en todos los niveles de autoestima excepto en la emocional. Se concluye cómo es necesario el soporte familiar para establecer patrones conductuales sanos en el adolescente.

PALABRAS CLAVE: ADOLESCENTE, AUTOESTIMA, FAMILIAR, BACHILLERATO, AUTOCONCEPTO.

\section{ABSTRACT}

School and family are a binomial linked to the academic performance and physical and emotional health of adolescents. It is in the school where they take great influence from their peers, resulting in the personality of the students; Therefore, self-esteem has a great impact as a psychological phenomenon. On the other hand, it is in the family that the first emotional bonds are established, greatly affecting the way adolescents live and interact. The objective of this study is to describe the relationship between self-esteem and family functionality in high school adolescents. A descriptive study was conducted to evaluate the family self-esteem and functionality of 349 second semester students of a public high school in the west of the Monterrey metropolitan area. The Form-5 (AF5) self-esteem scale and the Family APGAR questionnaire were used with support from the google forms platform. The application was collective within the university pre-authorization and consent. The results reveal in the majority of adolescents family functionality; in addition to significant correlation in levels of self-esteem; Except in the emotional. It is concluded how family support is necessary to establish healthy behavioral patterns in adolescents.

KEYWORDS: ADOLESCENT, SELF-ESTEEM, HIGH SCHOOL, FAMILY, HIGH SCHOOL, SELF-CONCEPT. 
$\mathrm{H}$ oy en día considerar el aspecto emocional en el ámbito educativo es una regulación enmarcada dentro de las acciones estratégicas prioritarias en todos los sectores (Secretaría de Educación Pública, 2018; OCDE, 2015; ANUIES, 2000). En este sentido, dirigir los esfuerzos en promoverlo dentro de las instituciones formativas en el Nivel de Educación Media Superior y Superior ha permitido contribuir en la mejora de habilidades cognitivas, afectivas, sociales y emocionales, sobre todo en la dinámica de convivencia y de aceptación entre estudiantes, profesores y, en general, de todos los implicados en el fenómeno educativo. De manera particular, aspectos como el autoconocimiento, autoconcepto y autoestima son recursos personales en donde se asume que el individuo percibe grados de logro, felicidad y satisfacción que inciden en el éxito académico (Seligman, 2011; Puigcerver, 2009; Vaello, 2007).

Definitivamente es en la escuela donde el adolescente toma gran influencia de sus pares dado el fenómeno de construir y redefinir su propia personalidad, por lo que el autoconcepto cobra gran impacto como fenómeno psicológico (Puigcerver, 2009). Ahora bien, la familia como agente de socialización es considerado uno de los factores más importantes en la construcción y desarrollo del autoconcepto en el adolescente, por lo que el contexto familiar enmarca factores protectores $\mathrm{y} / 0$ de riesgo en la promoción de la salud emocional y mental, y es base de la autoestima (Gutiérrez-Saldaña, Campacho-Calderón y Martínez-Martínez, 2007). Y sumados a la influencia del ambiente escolar adquieren un rol

\section{El grado de aceptabilidad social está grandemente influenciado por esa conceptualización de su propio comportamiento, además de tener gran influencia sobre las actitudes, sentimientos y conocimientos acerca de sus propias capacidades, habilidades y apariencia}

$\infty<\infty<\infty<\infty<\infty<\infty<\infty<\infty<\infty<\infty<\infty<\infty<\infty<\infty<\infty<\infty<\infty<\infty<\infty<\infty<\infty<\infty<\infty<\infty<\infty<\infty<\infty$

complementario para el estudiante de bachillerato. Enseguida se describen las implicaciones teóricas, así como la importancia del problema en el ámbito universitario, específicamente en Educación Media Superior.

\section{PLANTEAMIENTO DEL PROBLEMA}

En los últimos años un gran número de investigadores han abordado desde diferentes perspectivas los problemas emocionales de los adolescentes. Las intervenciones van desde el análisis de las variables involucradas en la salud mental, emocional y física de los estudiantes hasta la búsqueda de intervenciones psicoeducativas. Extremera y Fernández (2004) han identificado varios aspectos emocionales que afectan en el contexto educativo en el área del ajuste psicológico: calidad en las relaciones interpersonales, rendimiento académico y consumo de sustancias adictivas.

Dadas las circunstancias antes mencionadas, Cava (1998) elaboró y evaluó un programa de interven- ción para aumentar la autoestima y la integración social en el ámbito educativo, por lo que el papel que tiene la escuela como promotor de competencias socioemocionales es inminente (Mikulic, Crespi y Radusky, 2015; y Wang, Hu y Wang, 2018). Sin embargo, conjugarlo con la participación de los padres fortalece el desarrollo psicológico del adolescente (Dhillon y Kanwar, 2015). En este sentido, es importante explorar áreas relacionadas con situaciones emocionales, familiares, escolares y sociales.

En la adolescencia, los jóvenes reconstruyen su autoconcepto, es decir, tratan de definirse como personas, estudiantes, amigos, y así en cada uno de los roles que tienen socialmente. El grado de aceptabilidad social está grandemente influenciado por esa conceptualización de su propio comportamiento, además de tener gran influencia sobre las actitudes, sentimientos y conocimientos acerca de sus propias capacidades, habilidades y apariencia (Alvarado, 2002 en Gravini, 2016; La Rosa, 1986 


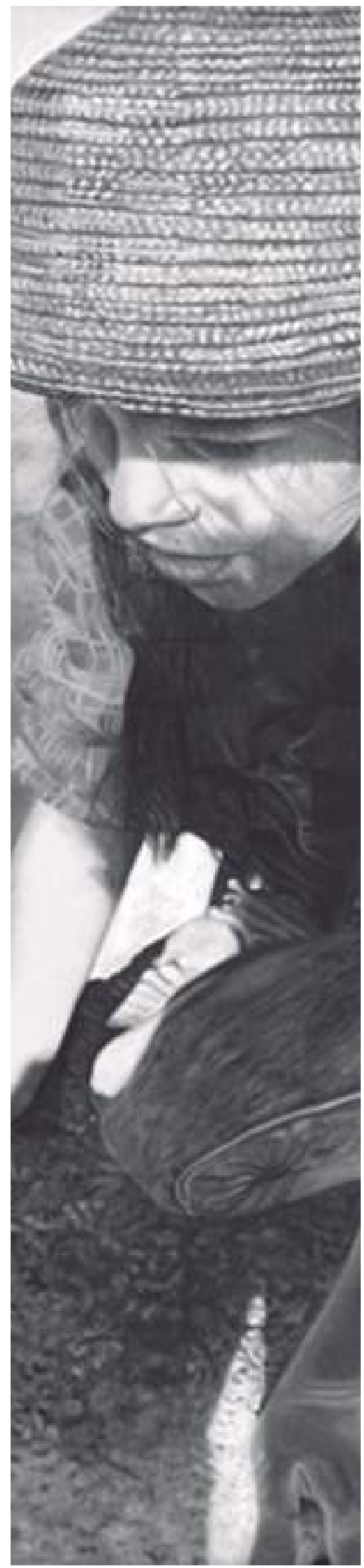

en Vera, Calderón y Rodríguez, 2016). Esta variable puede estar relacionada con la funcionalidad familiar, es decir, el rendimiento académico está asociado con expectativas de los padres respecto a sus hijos. De hecho, se ha encontrado una relación significativa entre la dinámica familiar y el rendimiento académico en niños y adolescentes, en diferentes regiones y clases sociales.

El objetivo general de la investigación es describir la relación entre autoestima y funcionalidad familiar en adolescentes de bachillerato.

Los objetivos específicos son los siguientes:

- Evaluar la autoestima de los estudiantes en sus 5 dimensiones: Académica, Social, Emocional, Familiar y Física.

- Evaluar el nivel de funcionalidad familiar que tienen los adolescentes.

- Establecer la relación entre el nivel de autoestima y funcionalidad familiar.

Y las hipótesis son las siguientes:

- Los niveles de autoestima son diferentes entre las familias funcionales y disfuncionales de adolescentes de bachillerato.
- La autoestima en sus 5 dimensiones (Académica, Social, Emocional, Familiar y Física) es significativamente diferente entre adolescentes con familias funcionales y disfuncionales.

\section{JUSTIFICACIÓN \\ DE LA INVESTIGACIÓN}

Hoy en día los índices de depresión e ideación suicida en adolescentes son una realidad social. Este hecho es paralelo al incremento de citas solicitadas en los Departamentos de Psicopedagogía en las escuelas y preparatorias a nivel regional en donde los adolescentes buscan ayuda sobre situaciones familiares, escolares y personales. Es necesario contar con un sistema de diagnóstico que permita reconocer variables emocionales y familiares que incida además en el rendimiento académico (Mikulic, Crespi, y Radusky, 2015).

Es fundamental también señalar cómo la autoestima y autoconcepto están asociados con el comportamiento agresivo, bullying, depresión y niveles de insatisfacción con la vida; por lo que valorar dichas variables se considera de impacto para la vida socioemocional de los adolescentes y sus familias (Andrade y González, 2017). de diagnóstico que permita reconocer variables emocionales y familiares que incida además en el rendimiento académico 


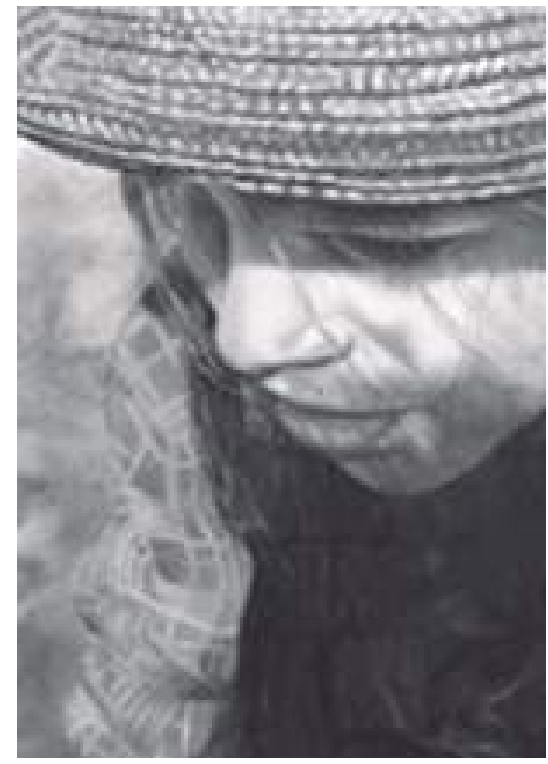

\section{AUTOESTIMA}

\section{Y AUTOCONCEPTO}

Se define como el conjunto de atributos que una persona utiliza para describirse a sí mismo y generalmente responde a la pregunta ¿Quién Soy? (Ruis, Contreras y Rivera, 1998; Paez, Zubieta, Mayordomo, Jiménez y Ruiz, 2004). También es considerada como una forma evaluativa que permite categorizar a la persona basado en las propias percepciones tanto positivas como negativas (Kanmani y Rajeswari, 2018) y son influidas por las experiencias y juicios que los demás asumen de uno mismo.Además, tiene carácter adaptativo ya que ayuda a la persona a guiar su comportamiento (Salum, Marín y Reyes, 2011). El Autoconcepto hace referencia a las áreas físicas, conductuales y afectivas de la persona (Bednar y Peterson, 1996). La acción valorativa del Autoconcepto se denomina Autoestima, pudiendo ser considerada como positiva 0 negativa (Urdiales, Castro y Villarreal, 2019).

\section{FUNCIONALIDAD FAMILIAR}

La familia es el primer vínculo que una persona posee y también la pri-

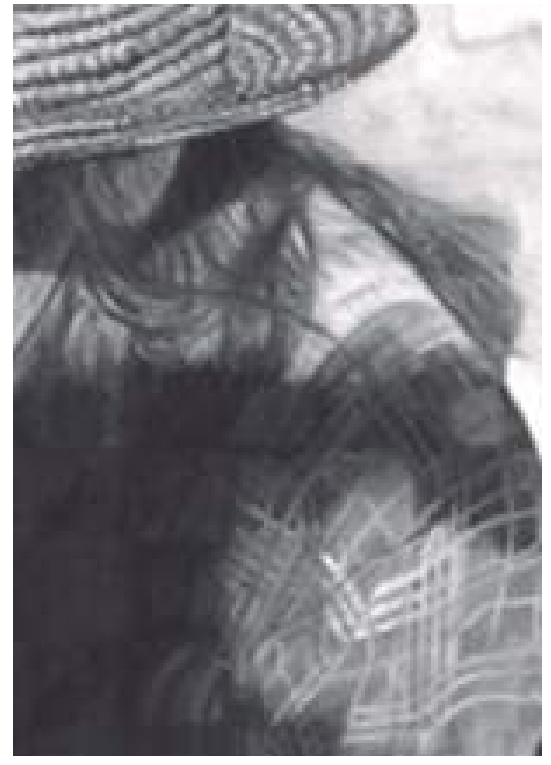

mera fuente de socialización en donde entran a efecto diferentes interacciones dentro de los subsistemas que lo conforman. Es en la familia donde se logra percibir un estado favorable de adaptabilidad, afecto, seguridad, participación y crecimiento, aunado a los recursos que se ofrecen para sentirse feliz, amado y vinculado positivamente (Moreno y Chauta, 2011).

Una familia funcional es capaz de realizar ajustes ante los cambios inminentes de la evolución del ciclo vital de la familia, afectando positiva o negativamente la vida de los individuos que lo conforman dependiendo de su grado de adaptabilidad. Por ejemplo, se ha encontrado relación entre adolescentes con problemas de alcohol 0 drogas en familias disfuncionales (Alonso-Castillo, Yáñez-Lozano, Armendariz-García, 2017).

\section{SELECCIÓN DE LA MUESTRA}

El estudio se realizó con un grupo de 349 adolescentes escolarizados de una preparatoria al poniente del área metropolitana de Monterrey. 152 hombres y 197 mujeres, todos de 2do semestre de bachillerato y con una media de 15 años. La muestra fue por conveniencia y como criterio de inclusión fue que aceptaran de forma voluntaria participar en la aplicación de los instrumentos.

\section{INSTRUMENTO \\ DE INVESTIGACIÓN}

La escala de autoestima Forma-5 (AF5) de García y Musitu (1999) es un instrumento de 30 ítems que mide 5 dimensiones dirigido a poblaciones mayores de 11 años. Es una escala tipo likert de 5 niveles que va de Nunca a Siempre, tiene un alpha de Cronbach de 0.81 y se ha validado en poblaciones de España, Italia, Brasil y Portugal.

Family APGAR es un cuestionario de 5 ítems que evalúa Adaptación, Paruna persona posee y también la primera fuente de socialización en donde entran a efecto diferentes interacciones dentro de los subsistemas que lo conforman 
ticipación Gradiente de Crecimiento, Afecto y Resolución. Se observa un solo factor explicando el $61.9 \%$ de la varianza, con validación en España. El índice de Kaiser-Mayer-Olkin fue de 0.82 y la prueba de esfericidad de Bartlett de 1.315 .2 ( $p<0.000050)$. La escala es tipo likert de 3 opciones de respuestas (Casi Nunca,A Veces y Casi Siempre). Si los valores son mayores a 6 se considera Funcional, mientras que valores obtenidos menores a 6 es Disfuncional.

\section{PROCEDIMIENTO}

Se procedió a realizar los permisos correspondientes para evaluar de manera colectiva a los estudiantes de bachillerato. Se obtuvo el consentimiento informado y se procedió a citar a los estudiantes en la sala de tecnología de la preparatoria. Con apoyo de la plataforma Google forms se hizo la aplicación de ambos instrumentos (Autoestima Forma-5 y Family APGAR). Una vez que se obtuvieron los datos se realizó el análisis de datos con apoyo del paquete estadístico SPSS.

\section{RESULTADOS}

Se ha obtenido el análisis descriptivo general de las características de las familias de los adolescentes. El $84 \%$ tienen familias nucleares, el $12 \%$ viven en familias de 3 generaciones (padres y abuelos) y el $3 \%$ en familias compuestas sólo por padre 0 madre. Por otro lado, el nivel de funcionalidad familiar en la población explorada revela que un $6 \%$ tienen nivel de disfuncionalidad grave, $16 \%$ disfuncionalidad leve y $78 \%$ perciben funcionalidad en su familia. Se realizó un primer análisis destacando correlación positiva entre los componentes de funcionalidad familiar

\section{La familia como fuente de socialización y soporte vital para el adolescente es un grupo de apoyo que puede facilitar el proceso de construcción del autoconcepto y la autoestima}

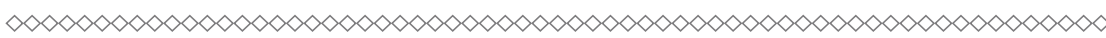

y los niveles de autoestima, exceptuando en la autoestima emocional.

\section{CONCLUSIONES Y PROPUESTAS}

\section{DE ACCIÓN Y TRANSFORMACIÓN}

La familia como fuente de socialización y soporte vital para el adolescente es un grupo de apoyo que puede facilitar el proceso de construcción del autoconcepto y la autoestima. Los resultados preliminares reflejan primacía en las características de funcionalidad presentes en la mayoría de las familias compuestas por padre, madre e hijos. Sin embargo, hay que hacer énfasis en el análisis en donde

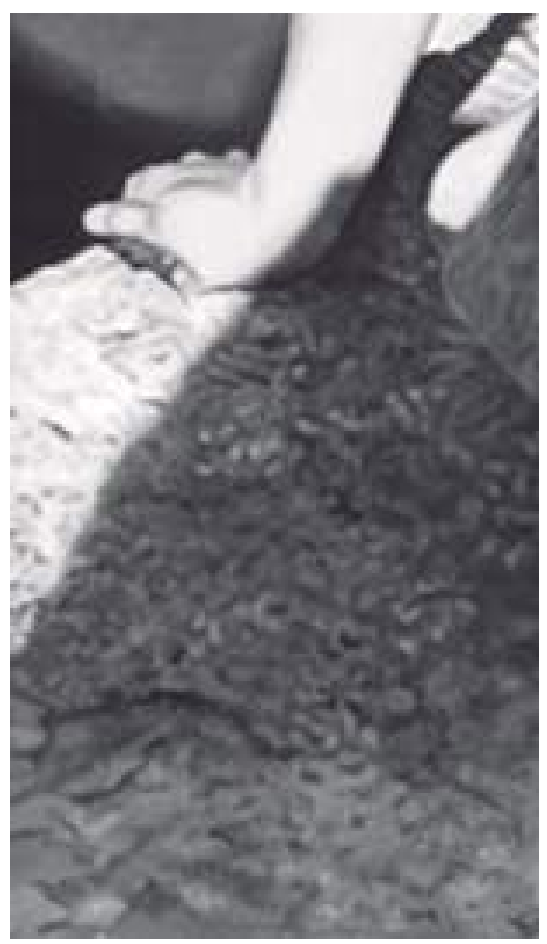

se agrupen por categorías los tipos de familia, el nivel de funcionalidad y los niveles de autoestima.

Como hallazgo preliminar se observa diferencia en la autoestima relativa al ajuste emocional y manejo del autocontrol (Autoestima Emocional). Sin embargo, es necesario hacer mayores análisis que destaquen dichas diferencias. Finalmente, es conveniente determinar con mayor validez y confiabilidad las características familiares asociadas con factores de riesgo o de protección en todos los adolescentes.

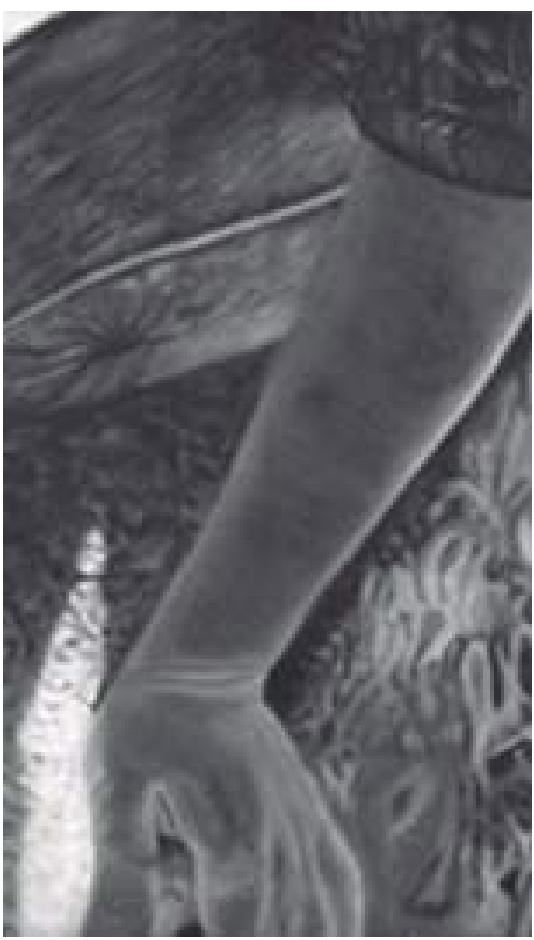




\section{REFERENCIAS}

Alonso-Castillo, M.; Yáñez-Lozano,A.; y Armendariz-García, N. (2017). Funcionalidad familiar y consumo de alcohol en adolescentes de secundaria. Healt and Addictions, 17(1), pp. 87-96. Andrade, J. y Gonzáles, J. (2017). Relación entre riesgo suicida, autoestima, desesperanza y estilos de socialización parental en estudiantes de bachillerato. Psicogente, 20(37), 70-88. Fuente: http://doi.org/10.17081/psico.20.37.2419.

ANUIES, (2000). La educación superior en el Siglo XXI: líneas de desarrollo, México, ANUIES. De: http://www.anuies.mx/servicios/d_estrategicos/documentos_estrategicos/21/sXXI.pdf.

Bednar, R.; y Peterson, S. (1996). Self-steem: paradoxes and innovations in clinical theory and practice. Washington, DC: American Psychological Association.

Extremera, N., y Fernández-Berrocal, P. (2004). El papel de la inteligencia emocional en el alumnado: evidencias empíricas. Revista Electrónica de Investigación Educativa, 6(2). Fuente: http://redie.uabc.mx/vol6no2/contenido-extremera.html.

Gravini, M.(2016). La permanencia y la deserción estudiantil y su relación con el autoconcepto, la resiliencia y el rendimiento académico en estudiantes con riesgo socioeconómico alto 0 bajo. Tesis doctoral. Universidad Autónoma de Madrid, Facultad de Formación de Profesorado y Educación. España.

Gutiérrez-Saldaña, P.; Campacho-Calderón, N.; y Martínez-Martínez, M. (2007). Autoestima, funcionalidad familiar y rendimiento escolar en adolescentes. Aten Primaria, 39(11), pp.597601.

Kanmani, M. y Rajeswari, N. (2018). A study on self-esteem among Tamil Graduate students Chennai. IJAR International Journal of Advanced Research. DOI: 10.21474/IJAR/7423.

OECD (2015). Skills for Social Progress: The Power of Social and Emotional Skills, OECD Skills Studies, OECD Publishing, Paris.

Mikulic, I.M., Crespi, M., y Radusky, P. (2015). Construcción y Validación del Inventario de Competencias Socioemocionales para Adultos (ICSE). Revista Interdisciplinaria, Universidad de Buenos Aires, 32(2), 307-330. Consultada el 17 de abril de 2018 en: http://www.redalyc. org/pdf/180/18043528007.pdf.

Moreno,J.; yChauta, L.(2011). Funcionalidad familiar, conductas externalizadas y rendimiento académico en un grupo de adolescentes de la ciudad de Bogotá. Psicologia: Avances de la disciplina, 6(1), pp. 155-166.

Puigcerver, M. (2009). Evaluación del Autoconcepto, Actitudes y Competencia Social en Sujetos Sordos, (Tesis Doctorales) Universidad de Murcia.

Paez, D.; Zubieta, E.; Mayordomo, S.; Jimenez, A.; y Ruiz, S. (2004). Identidad, autoconcepto, auto-estima y autoeficacia y locus de control. ISBN 84-205-3724-1, págs. 125-194.

Ruis M., Contreras M., y Ribera B., (1998). Aportaciones sistémicas sobre autoestima y autoconcepto en Educación para la Salud. Fuente: https://zenodo.org/record/1069806/ files/4\%20Rius.pdf.

Salum, A.; Marín, R.; y Reyes, C. (2011). Relevancia de las dimensiones del autoconcepto en estudiantes de secundaria de ciudad Victoria, Tamaulipas; México. Revista Electrónica de Psicología Iztacala. 14, (2) pp. 255-272.

Seligman, M. (2011). Florecer: psicología; psicología positiva; sentido de la felicidad; bienestar. Océano exprés.

SEP (2018). Nuevo Modelo Educativo, Escuelas Normales. Resumen Ejecutivo. Fuente: https:// docs.google.com/gview?url=https://www.gob.mx/cms/uploads/attachment/file/240634/1.-_ Resumen_Ejecutivo_7_.pdf.

Urdiales, M.; Castro,C.; Villarreal, M. (2018). El autoesquema y el autoconcepto en el ámbito escolar. En Martínez, Y.; Aceves, Y.; y Barak, M. (2018). Desarrollo del Potencial Humano en el ámbito universitario. Artificios Editorial, Universidad Autónoma de Baja California, pp.19-41.

Vaello, J. (2007). Cómo dar clase a los que no quieren. Santillana pp.45-53.

Vera, J., Calderón, N. y Rodríguez C. (2016). El ajuste psicosocial de alumnos de nuevo ingreso y la atención tutorial en la universidad. Psychosocial adjustment of students new and attention the tutorial at the university, 8(14).

Wang, J; Hu, S; y Wang, L. (2018). Multilevel analysis of personality, family, and classroom influences on emotional and behavioral problems among.

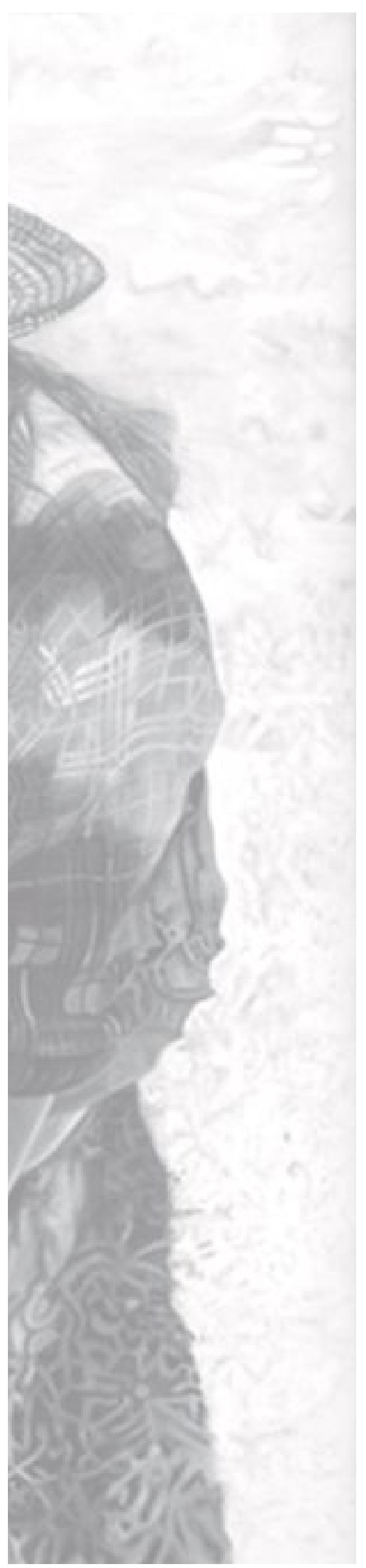




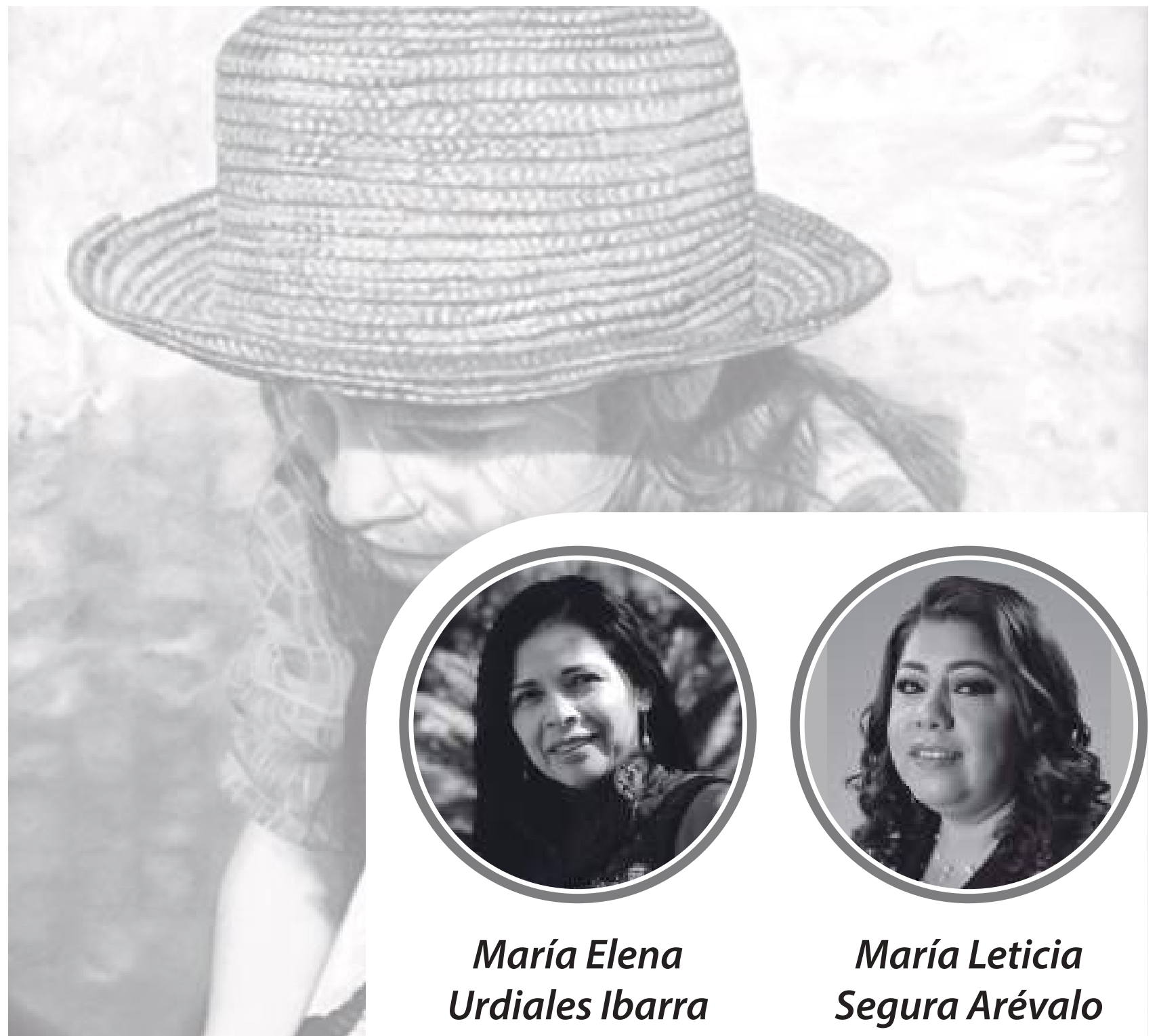

Es Doctora en Filosofía con especialidad en Psicología egresada con distinción Cum Laude. Es profesora de tiempo completo de la Facultad de Psicología de la Universidad Autónoma de Nuevo León. Es investigadora perteneciente al Cuerpo Académico de Ciencia Cognitiva de la Facultad de Psicología en la UANL. Es evaluadora de programas de Psicología por el Comité Acreditador del Consejo Nacional de Enseñanza e Investigación en Psicología.

Correo Electrónico: malena_urdiales@hotmail.com
Es estudiante del Doctorado en Psicología Educativa con Maestría en Docencia con Orientación en el Nivel Medio Superior. Profesora de tiempo completo de la Preparatoria 7 de la UANL. Integrante de la Comisión Legislativa del Honorable Consejo Universitario de la UANL. Facilitador certificado de Mecanismos Alternativos para el ejercicio de la Mediación y Conciliación en el Estado de Nuevo León. Actualmente es directora de la Preparatoria 7 de la UANL.

\section{Correo Electrónico:}

lettysegura@hotmail.com

Recibido: 15/09/2019

Aceptado: 15/10/2019 